PROCEEDINGS OF THE

AMERICAN MATHEMATICAL SOCIETY

Volume 137, Number 4, April 2009, Pages 1307-1313

S 0002-9939(08)09661-5

Article electronically published on November 6, 2008

\title{
ADMISSIBLE LOCAL SYSTEMS FOR A CLASS OF LINE ARRANGEMENTS
}

\author{
SHAHEEN NAZIR AND ZAHID RAZA
}

(Communicated by Alexander N. Dranishnikov)

\begin{abstract}
A rank one local system $\mathcal{L}$ on a smooth complex algebraic variety $M$ is admissible if roughly speaking the dimension of the cohomology groups $H^{m}(M, \mathcal{L})$ can be computed directly from the cohomology algebra $H(M, \mathbb{C})$.

We say that a line arrangement $\mathcal{A}$ is of type $\mathcal{C}_{k}$ for some $k \geq 0$ if $k$ is the minimal number of lines in $\mathcal{A}$ containing all the points of multiplicity at least 3. We show that if $\mathcal{A}$ is a line arrangement in the classes $\mathcal{C}_{k}$ for $k \leq 2$, then any rank one local system $\mathcal{L}$ on the line arrangement complement $M$ is admissible. Partial results are obtained for the class $\mathcal{C}_{3}$.
\end{abstract}

\section{INTRODUCTION}

When $M$ is a hyperplane arrangement complement in some projective space $\mathbb{P}^{n}$, one defines the notion of an admissible local system $\mathcal{L}$ on $M$ in terms of some conditions on the residues of an associated logarithmic connection $\nabla(\alpha)$ on a good compactification of $M$; see for instance [7, [11, 8], 9], 6]. This notion plays a key role in the theory, since for such an admissible local system $\mathcal{L}$ on $M$ one has

$$
\operatorname{dim} H^{i}(M, \mathcal{L})=\operatorname{dim} H^{i}\left(H^{*}(M, \mathbb{C}), \alpha \wedge\right)
$$

for all $i \in \mathbb{N}$.

Let $\mathcal{A}$ be a line arrangement in the complex projective plane $\mathbb{P}^{2}$ and denote by $M$ the corresponding arrangement complement. For the case of line arrangements, a good compactification is obtained just by blowing-up the points of multiplicity at least 3 in $\mathcal{A}$. This explains the simple version of the admissibility definition given below in Definition 2.1,

Let $k$ be a non-negative integer. We say that a line arrangement $\mathcal{A}$ is of type $\mathcal{C}_{k}$ if $k$ is the minimal number of lines in $\mathcal{A}$ containing all the points of multiplicity at least 3. For instance, $k=0$ corresponds to nodal arrangements, while $k=1$ corresponds to the case of a nodal affine arrangement; see [1]. Note that $k=k(\mathcal{A})$ is combinatorially defined; i.e. it depends only on the intersection lattice $L(\mathcal{A})$.

The main result of this note is the following one, which, as explained above, is new in the case $n=2$.

Theorem 1.1. Let $\mathcal{A}$ be a line arrangement in $\mathbb{P}^{2}$. If $\mathcal{A}$ belongs to the class $\mathcal{C}_{k}$ for some $k \leq 2$, then any rank one local system $\mathcal{L}$ on $M$ is admissible.

Received by the editors January 22, 2008, and, in revised form, June 2, 2008.

2000 Mathematics Subject Classification. Primary 14C21, 14F99, 32S22; Secondary 14E05, $14 \mathrm{H} 50$.

Key words and phrases. Admissible local system, line arrangement, characteristic variety.

(C) 2008 American Mathematical Society 1307

Reverts to public domain 28 years from publication 
This result implies in particular that for a combinatorially defined class of arrangements, the characteristic varieties are determined by $L(\mathcal{A})$; see Theorem 2.2

In section 2 we first make explicit the admissibility condition in the case of line arrangements. Then we prove our main theorem and derive some consequences for the characteristic varieties of line arrangements in the classes $\mathcal{C}_{k}$ for $k \leq 2$.

In the final section we find some sufficient conditions for a local system $\mathcal{L}$ on the complement of a line arrangement in the class $\mathcal{C}_{3}$ to be admissible; see Proposition 3.1

The deleted $B_{3}$-arrangement discovered by A. Suciu shows that Theorem 1.1 does not hold for $k=3$, so our result is the best possible. A discussion of this example from the point of view of our paper is given in Example 3.2. Further examples conclude the paper.

\section{Admissible RANK ONE LOCAL SYSTEMS}

Let $\mathcal{A}=\left\{L_{0}, L_{1}, \ldots, L_{n}\right\}$ be a line arrangement in $\mathbb{P}^{2}$ and set $M=\mathbb{P}^{2} \backslash\left(L_{0} \cup\right.$ $\left.\ldots \cup L_{n}\right)$. Let $\mathbb{T}(M)=\operatorname{Hom}\left(\pi_{1}(M), \mathbb{C}^{*}\right)$ be the character group of $M$. This is an algebraic torus $\mathbb{T}(M) \simeq\left(\mathbb{C}^{*}\right)^{n}$. Consider the exponential mapping

$$
\exp : H^{1}(M, \mathbb{C}) \rightarrow H^{1}\left(M, \mathbb{C}^{*}\right)=\mathbb{T}(M)
$$

induced by the usual exponential function $\mathbb{C} \rightarrow \mathbb{C}^{*}, t \mapsto \exp (2 \pi i t)$, where $i=\sqrt{-1}$. Clearly $\exp \left(H^{1}(M, \mathbb{C})\right)=\mathbb{T}(M)$ and $\exp \left(H^{1}(M, \mathbb{Z})\right)=\{1\}$.

A rank one local system $\mathcal{L} \in \mathbb{T}(M)$ is identified with the corresponding homomorphism $\rho: \pi_{1}(M) \rightarrow \mathbb{C}^{*}$. This homomorphism is determined by the induced morphism $\tilde{\rho}: H_{1}(M, \mathbb{Z}) \rightarrow \mathbb{C}^{*}$. The homology group $H_{1}(M, \mathbb{Z})$ is generated by the elementary loops $\gamma_{j}$ around the lines $L_{j}$, with a single relation, namely $\gamma_{0}+\ldots+\gamma_{n}=0$; see [10. If we set $\lambda_{j}=\tilde{\rho}\left(\gamma_{j}\right)$, then $\lambda_{0} \ldots \lambda_{n}=1$. A cohomology class $\alpha \in H^{1}(M, \mathbb{C})$ is given by

$$
\alpha=\sum_{j=0}^{n} a_{j} \frac{d f_{j}}{f_{j}}
$$

with the residues $a_{j} \in \mathbb{C}$ satisfying $\sum_{j=0}^{n} a_{j}=0$ and $f_{j}=0$ being a linear equation for the line $L_{j}$. With this notation one has $\exp (\alpha)=\mathcal{L}$ if and only if $\lambda_{j}=\exp \left(2 \pi i a_{j}\right)$ for $j=0, \ldots, n$.

Definition 2.1. A local system $\mathcal{L} \in \mathbb{T}(M)$ as above is admissible if there is a cohomology class $\alpha \in H^{1}(M, \mathbb{C})$ as in $(2.2)$ such that $\exp (\alpha)=\mathcal{L}, a_{j} \notin \mathbb{Z}_{>0}$ and, for any point $p \in L_{0} \cup \ldots \cup L_{n}$ of multiplicity at least 3 , one has

$$
a(p)=\sum_{j} a_{j} \notin \mathbb{Z}_{>0}
$$

where the sum is over all $j$ 's such that $p \in L_{j}$.

For an admissible local system the isomorphisms in (1.1) were shown in [7, 11].

Proof of Theorem 1.1. We can assume that, for a line arrangement $\mathcal{A}$ of type $\mathcal{C}_{k}$, the lines in $\mathcal{A}$ containing all the points of multiplicity at least 3 are $L_{0}, \ldots, L_{k-1}$. Set $b(p)=\Re(a(p))$, where $\Re$ denotes the real part of a complex number.

Given $\mathcal{L}$, for the residues $a_{j} \in \mathbb{C}$ we can choose $\Re\left(a_{j}\right) \in[0,1)$ for all $j \geq 1$. Then the relation $\sum_{j=0}^{n} a_{j}=0$ implies that 


$$
\Re\left(a_{0}\right)=-\sum_{j=1}^{n} \Re\left(a_{j}\right) \leq 0 .
$$

This completes the proof in the case where $\mathcal{A}$ is in the class $\mathcal{C}_{0}$.

Consider now the case where $\mathcal{A}$ is in the class $\mathcal{C}_{1}$ and choose $p \in L_{0}$ as in Definition 2.1. If the set of lines passing through $p$ is $L_{0}, L_{j_{1}}, \ldots, L_{j_{s}}$ for some $s \geq 2$, then one has

$$
b(p)=\sum_{j} \Re\left(a_{j}\right)=-\sum_{s} \Re\left(a_{s}\right) \leq 0
$$

where the second sum is over all $s>0$ such that $p \notin L_{j_{s}}$. This shows that any local system $\mathcal{L} \in \mathbb{T}(M)$ is admissible in this case.

The case where $\mathcal{A}$ is in the class $\mathcal{C}_{2}$ is much more subtle. Let $m_{1}$ be the maximum of the real numbers $b(p)$ where $p$ is a point of multiplicity at least 3 on the line $L_{1}$ but not on $L_{0}$ and $b(p) \in \mathbb{Z}_{>0}$. By convention we set $\max \emptyset=0$.

If $m_{1}=0$, then the initial choice is good, exactly as in the previous situation.

Assume now that $m_{1}>0$ and let $p_{1} \in L_{1}$ be a multiple point with $b\left(p_{1}\right)=m_{1}$. In general this value $m_{1}$ can be attained at several points $p_{1}, p_{1}^{\prime}, p_{1}^{\prime \prime}, \ldots$ on $L_{1}$. Any such point will be called an extremal point for $\mathcal{L}$ on $L_{1}$.

Then we replace $a_{1}$ by $a_{1}^{\prime}=a_{1}-m_{1}$, and hence $\Re\left(a_{1}^{\prime}\right) \leq 0$ if $\Re\left(a_{1}^{\prime}\right)$ is an integer. We also replace $a_{0}$ by $a_{0}^{\prime}=a_{0}+m_{1}$, so that the total sum of the residues is still zero. Note that $\Re\left(a_{0}^{\prime}\right) \leq 0$.

After these two changes, it is clear that $b(q) \notin \mathbb{Z}_{>0}$ for any point $q$ which is of multiplicity at least 3 on $L_{1}$ but not on $L_{0}$. If $O=L_{0} \cap L_{1}$ is a point of multiplicity at least 3 , then

$$
b(O)=\Re\left(a_{0}^{\prime}\right)+\Re\left(a_{1}^{\prime}\right)+\sum_{m} \Re\left(a_{m}\right)=-\sum_{t} \Re\left(a_{t}\right) \leq 0
$$

where the first sum is over $m=2, \ldots, n$ such that $O \in L_{m}$, and the second sum is over all $t=2, \ldots, n$ such that $O \notin L_{t}$. Note that $O$ is never an extremal point.

If now $q \neq O$ is a point of multiplicity at least 3 on the line $L_{0}$, in the corresponding sum for $b(q)$ we have the following terms:

(i) $-\Re\left(a_{j}\right)$ for all $j>0$,

(ii) $+\Re\left(a_{j_{t}}\right)$ for all the lines different from $L_{0}$ passing through $q$,

(iii) $+\Re\left(a_{j_{s}}\right)$ for all the lines passing through $p_{1}$.

(In fact the terms of type (i) come from $\Re\left(a_{0}\right)$, and the terms of type (iii) come from adding $m_{1}$ to $a_{0}$ to get $a_{0}^{\prime}$.)

In the last two families there is at most one line in common, namely the line determined by $p_{1}$ and $q$. It follows that the sum for $b(q)$ involves some negative terms from (i) and possibly one positive term in the interval $[0,1)$. Therefore $b(q)<1$, and this concludes the proof of Theorem 1.1.

The characteristic varieties of $M$ are the jumping loci for the cohomology of $M$, with coefficients in rank 1 local systems:

$$
\mathcal{V}_{d}^{i}(M)=\left\{\rho \in \mathbb{T}(M) \mid \operatorname{dim} H^{i}\left(M, \mathcal{L}_{\rho}\right) \geq d\right\} .
$$

When $i=1$, we use the simpler notation $\mathcal{V}_{d}(M)=\mathcal{V}_{d}^{1}(M)$. One has the following result; see [4, Remark 2.9 (ii).

Theorem 2.2. If any local system in $\mathbb{T}(M)$ is admissible, then for any $d$ there are no translated components in the characteristic variety $\mathcal{V}_{d}(M)$. 
In particular, for any irreducible component $W$ of some characteristic variety $\mathcal{V}_{d}(M)$, the dimension of $H^{1}(M, \mathcal{L})$ is constant for $\mathcal{L} \in W \backslash\{1\}$.

Combining this result and our Theorem 1.1, we get the following.

Corollary 2.3. If $\mathcal{A}$ is a line arrangement in the classes $\mathcal{C}_{k}$ for $k \leq 2$, then for any $d$ there are no translated components in the characteristic variety $\mathcal{V}_{d}(M)$.

\section{Line arRangements in the Class $\mathcal{C}_{3}$}

In this section we discuss only the situation where the 3 lines $L_{0}, L_{1}$ and $L_{2}$ containing all the points of multiplicity at least 3 are concurrent. Let $O=L_{0} \cap$ $L_{1} \cap L_{2}$. Let $\mathcal{L} \in \mathbb{T}(M)$ be a rank one local system and choose the residues $a_{j}$ for $0 \leq j \leq n$ as above. Then we have a collection $P_{1}$ of extremal points for $\mathcal{L}$ on $L_{1}$ and a collection $P_{2}$ of extremal points for $\mathcal{L}$ on $L_{2}$. By convention $P_{j}$ is empty if the corresponding maximum $m_{j}=0$ (see also the beginning of the proof below for the definition of $\left.m_{j}\right)$. Since we start with the $a_{j}$ 's such that $\Re\left(a_{j}\right) \in[0,1)$ for all $j>0$, we have $O \notin P_{1} \cup P_{2}$ as above. For each point $q \in L_{0}$ of multiplicity at least 3 , we denote by $\mathcal{A}_{q}$ the set of lines in $\mathcal{A}$ passing through $q$.

Proposition 3.1. With the above notation, assume that one of the following conditions holds:

(i) $P_{1}=\emptyset$;

(ii) $P_{2}=\emptyset$;

(iii) for each point $q \in L_{0}$ of multiplicity at least 3, one has either

(a) $P_{1} \backslash Y_{q}$ and $P_{2} \backslash Y_{q}$ are both non-empty, where $Y_{q}$ is the union of the lines in $\mathcal{A}_{q}$, or

(b) one of the sets $P_{1} \backslash Y_{q}$ and $P_{2} \backslash Y_{q}$ is non-empty, say $p_{1} \in P_{1} \backslash Y_{q}$, and there is an extremal point $p_{2} \in P_{2}$ such that the line determined by $p_{1}$ and $p_{2}$ is not in $\mathcal{A}$.

Then the local system $\mathcal{L}$ is admissible.

Proof. Let $m_{j}$, for $j=1,2$, be the maximum of the real numbers $b(p)$ where $p \neq O$ is a point of multiplicity at least 3 on the line $L_{j}$ such that $b(p) \in \mathbb{Z}_{>0}$. The only essentially new case is when $m_{1}>0$ and $m_{2}>0$, i.e. case (iii) above. Then we set $a_{j}^{\prime}=a_{j}-m_{j}$ for $j=1,2$ and $a_{0}^{\prime}=a_{0}+m_{1}+m_{2}$. Exactly as in the proof of Theorem 1.1 this settles the case of multiple points on $L_{1}$ and $L_{2}$ distinct from $O$. At the point $O$ we get the sum

$$
b(O)=\Re\left(a_{0}^{\prime}+a_{1}^{\prime}+a_{2}^{\prime}\right)+\sum_{m} \Re\left(a_{m}\right)=-\sum_{t} \Re\left(a_{t}\right) \leq 0
$$

where the first sum is over $m=3, \ldots, n$ such that $O \in L_{m}$, and the second sum is over all $t=3, \ldots, n$ such that $O \notin L_{t}$.

If now $q \neq O$ is a point of multiplicity at least 3 on the line $L_{0}$, in the corresponding sum for $b(q)$ we have the following terms:

(i) $-\Re\left(a_{j}\right)$ for all $j>0$;

(ii) $+\Re\left(a_{j_{s}}\right)$ for all the lines different from $L_{0}$ passing through $q$;

(iii) $+\Re\left(a_{j_{m}}\right)$ for all the lines passing through $p_{1}$, for a fixed choice $p_{1} \in P_{1}$;

(iv) $+\Re\left(a_{j_{r}}\right)$ for all the lines passing through $p_{2}$, for a fixed choice $p_{2} \in P_{2}$.

(In fact the terms of type (i) come from $\Re\left(a_{0}\right)$, the terms of type (iii) come from adding $m_{1}$, and the terms of type (iv) come from adding $m_{2}$.) 
Our assumption (iii) says exactly that there is a choice of $p_{1}$ and $p_{2}$ such that in the last 3 families of positive terms there is at most one line occurring twice (and none occurring three times). It follows that the sum for $b(q)$ involves some negative terms from (i) and possibly one positive term in the interval $[0,1)$. Therefore $b(q)<1$, and this concludes the proof.

Example 3.2. Here we analyze from the point of view of the above proof the non-admissible local systems in the case of Suciu's arrangement 12 .

Choose: $L_{0}: x=0, L_{1}: x=y, L_{2}: y=0, L_{3}: x=z, L_{4}: y=z$, $L_{5}: x-y+z=0, L_{6}: x-y-z=0$ and $L_{7}: z=0$ (this last one is the line at infinity).

There are 7 points of multiplicity at least 3, namely, $O=L_{0} \cap L_{1} \cap L_{2}=(0$ : $0: 1), p_{1}=L_{1} \cap L_{3} \cap L_{4}=(1: 1: 1), p_{1}^{\prime}=L_{1} \cap L_{5} \cap L_{6} \cap L_{7}=(1: 1: 0)$, $p_{2}=L_{2} \cap L_{3} \cap L_{6}=(1: 0: 1), p_{2}^{\prime}=L_{2} \cap L_{4} \cap L_{7}=(1: 0: 0), q_{1}=L_{0} \cap L_{4} \cap L_{5}$ and $q_{2}=L_{0} \cap L_{3} \cap L_{7}=(0: 1: 0)$. It is clear that $L_{0}, L_{1}, L_{2}$ contain all the points of multiplicity at least 3 , even those at infinity.

Consider the local system $\mathcal{L}_{\rho}$ which is used to define the translated component

$$
W=\rho \cdot\left\{\left(t, 1, t^{-1}, t^{-1}, t, t^{-2}, t^{2}, 1\right) \mid t \in \mathbb{C}^{*}\right\},
$$

where $\rho=(1,-1,-1,-1,1,1,1,-1)$. The corresponding residues are $a_{1}=a_{2}=$ $a_{3}=a_{7}=1 / 2, a_{4}=a_{5}=a_{6}=0$ and $a_{0}=-2$. Using these residues we find out that $m_{1}=m_{2}=1, P_{1}=\left\{p_{1}, p_{1}^{\prime}\right\}$ and $P_{2}=\left\{p_{2}, p_{2}^{\prime}\right\}$.

For $q=q_{2}$, both sets $P_{1} \backslash Y_{q}$ and $P_{2} \backslash Y_{q}$ are empty. Thus condition (iii) of Proposition 3.1 is not fulfilled.

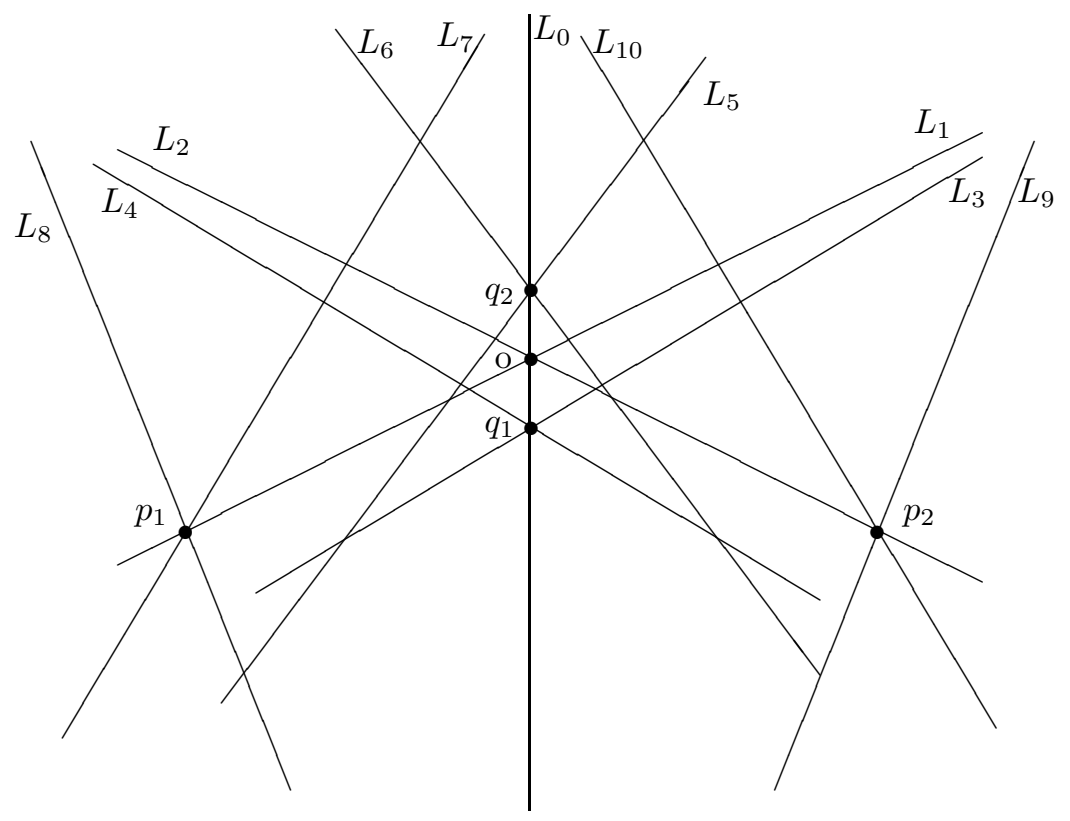

FIGURE 1 
Example 3.3. Here is an example of an arrangement in $\mathcal{C}_{3}$ of the type discussed above for which all local systems are admissible. Let $\mathcal{A}$ be the arrangement in $\mathbb{C}^{2}$ consisting of the following 10 lines: $L_{0}: x=0, L_{1}: y=1 / 2(x+3), L_{2}: y=$ $-1 / 2(x-3), L_{3}: y=x+1, L_{4}: y=-(x-1), L_{5}: y=2(x+1), L_{6}: y=$ $-2(x-1), L_{7}: y=3 / 2(x+3), L_{8}: y=-3 / 2(x+3), L_{9}: y=5 / 2(x-3)$ and $L_{10}: y=-5 / 2(x-3)$.

There are 5 triple points as shown in Fig. $1 ; 3$ are on the line $L_{0}$, namely $O=L_{1} \cap L_{2} \cap L_{0}=\left(0, \frac{3}{2}\right), q_{1}=L_{0} \cap L_{3} \cap L_{4}=(0,1)$ and $q_{2}=L_{0} \cap L_{5} \cap L_{6}=(0,2)$; and the other 2 are $p_{1}=L_{1} \cap L_{7} \cap L_{8}=(-3,0)$ and $p_{2}=L_{2} \cap L_{9} \cap L_{10}=(3,0)$. Any local system is admissible by Proposition 3.1. Indeed, if we suppose that $P_{1}$ and $P_{2}$ are both non-empty, then both sets $P_{1} \backslash Y_{q}=P_{1}$ and $P_{2} \backslash Y_{q}=P_{2}$ are again non-empty, for $q=q_{1}$ and $q=q_{2}$.

Example 3.4. Now we present an example of an arrangement in $\mathcal{C}_{3}$ of the type discussed above for which Proposition 3.1 does not apply, but where nevertheless most (perhaps all) local systems are admissible. Let $\mathcal{A}$ be the arrangement in $\mathbb{C}^{2}$ consisting of the following 7 lines: $L_{0}: x=0, L_{1}: y=-2(x-1), L_{2}: y=2(x+1)$, $L_{3}: y=-(x-1), L_{4}: y=x+1, L_{5}: y=-1 / 3(x+1)$ and $L_{6}: y=1 / 3(x-1)$. The line at infinity is denoted by $L_{7}$.

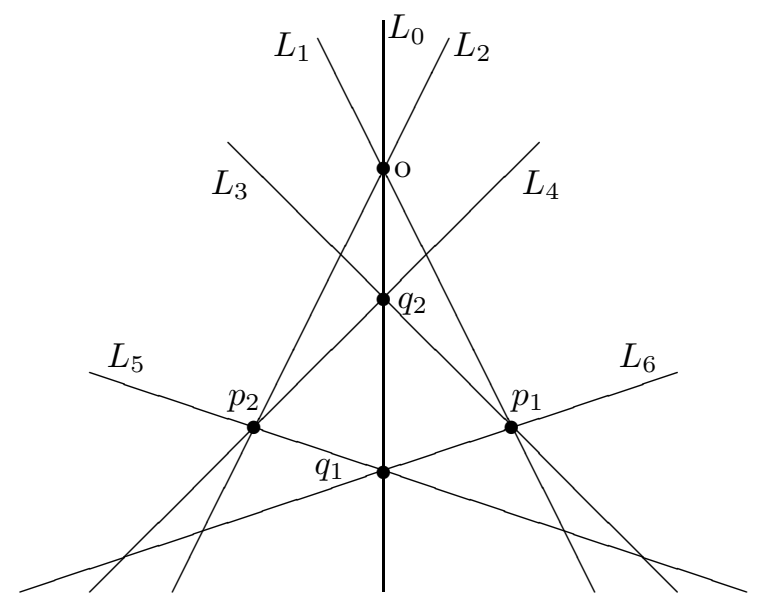

FIGURE 2

There are 5 points of multiplicity at least 3 ; see Fig. 2. These are $O=L_{0} \cap$ $L_{1} \cap L_{2}=(0,2), p_{1}=L_{1} \cap L_{3} \cap L_{6}=(0,1), p_{2}=L_{2} \cap L_{4} \cap L_{5}=(-1,0)$, $q_{1}=L_{0} \cap L_{5} \cap L_{6}=\left(0,-\frac{1}{3}\right)$ and $q_{2}=L_{0} \cap L_{3} \cap L_{4}=(0,1)$. In fact all these points have multiplicity 3 and lie on either $L_{0}, L_{1}$ or $L_{2}$.

Assume that we have a local system $\mathcal{L}$ such that $P_{1}$ and $P_{2}$ are both non-empty. Then $m_{1}=\Re\left(a_{2}+a_{4}+a_{5}\right) \in\{1,2\}$ and $m_{2}=\Re\left(a_{1}+a_{3}+a_{6}\right) \in\{1,2\}$. In this case, the new residue along $L_{0}$ is

$$
a_{0}^{\prime}=a_{0}+m_{1}+m_{2}=-\sum_{j=1}^{7} a_{j}+m_{1}+m_{2}=-a_{7}-i \cdot \Im\left(\sum_{j=1}^{6} a_{j}\right)
$$

where $\Im$ denotes the imaginary part of a complex number. When we compute the residue along the exceptional curve $E_{1}$ created by blowing-up the point $q_{1}$, we get 


$$
r_{1}=a_{0}^{\prime}+a_{5}+a_{6}=a_{5}+a_{6}-a_{7}-i \cdot \Im\left(\sum_{j=1}^{6} a_{j}\right) .
$$

Similarly we get

$$
r_{2}=a_{3}+a_{4}-a_{7}-i \cdot \Im\left(\sum_{j=1}^{6} a_{j}\right)
$$

for the point $q_{2}$. In this case Proposition 3.1 does not apply, since for $q=q_{1}, q_{2}$ both sets $P_{1} \backslash Y_{q}$ and $P_{2} \backslash Y_{q}$ are empty. However, whenever $\Re\left(r_{1}\right)$ and $\Re\left(r_{2}\right)$ do not belong to $\mathbb{Z}_{>0}$, the corresponding local system $\mathcal{L}$ is admissible.

\section{REFERENCES}

1. A.D.R. Choudary, A. Dimca, S. Papadima: Some analogs of Zariski's Theorem on nodal line arrangements, Algebraic and Geometric Topology 5(2005), 691-711. MR2153112 (2006f:32038)

2. D.C. Cohen, A.I. Suciu: Characteristic varieties of arrangements, Math. Proc. Cambridge Philos. Soc. 127(1999), 33-54. MR.1692519 (2000m:32036)

3. A. Dimca: Sheaves in Topology, Universitext, Springer-Verlag, Berlin, 2004. MR2050072 (2005j:55002)

4. A. Dimca: On admissible rank one local systems, J. Algebra (2008), doi:10.1016/j.jalgebra.2008.01.039.

5. A. Dimca, S. Papadima, A. Suciu: Formality, Alexander invariants, and a question of Serre, math.AT/0512480.

6. A. Dimca, L. Maxim: Multivariable Alexander invariants of hypersurface complements, Trans. Amer. Math. Soc. 359(2007), no. 7, 3505-3528. MR2299465

7. H. Esnault, V. Schechtman, E. Viehweg: Cohomology of local systems on the complement of hyperplanes, Invent. Math. 109(1992), 557-561. Erratum, ibid. 112(1993), 447. MR1176205 (93g:32051) MR:1213111 (94b:32061)

8. M. Falk: Arrangements and cohomology, Ann. Combin. 1(1997), no. 2, 135-157. MR1629681 (99g:52017)

9. A. Libgober, S. Yuzvinsky: Cohomology of the Orlik-Solomon algebras and local systems, Compositio Math. 121(2000), 337-361. MR1761630(2001j:52032)

10. P. Orlik, H. Terao: Arrangements of hyperplanes, Springer-Verlag, Berlin, 1992. MR1217488 (94e:52014)

11. V. Schechtman, H. Terao, A. Varchenko: Local systems over complements of hyperplanes and the Kac-Kazhdan condition for singular vectors, J. Pure Appl. Alg. 100(1995), no. 1-3, 93-102. MR 1344845 (96j:32047)

12. A. Suciu: Translated tori in the characteristic varieties of complex hyperplane arrangements. Arrangements in Boston: A Conference on Hyperplane Arrangements (1999). Topology Appl. 118(2002), no. 1-2, 209-223. MR1877726 (2002j:32027)

Abdus Salam School of Mathematical Sciences, Government College University, 68-B New Muslim Town, Lahore, Pakistan

Current address: Abdus Salam School of Mathematical Sciences, Government College University, 35 C-2 Gulberg III, Lahore, Pakistan

E-mail address: shaheen.nazeer@gmail.com

Abdus Salam School of Mathematical Sciences, Government College University, 68-B New Muslim Town, Lahore, Pakistan

E-mail address: zahidsms@gmail.com 